\title{
Erratum and Comment: Topological Spin-Statistics Relation in Quantum Field Theory ${ }^{1}$
}

\author{
R. D. Tscheuschner ${ }^{2}$
}

There is a misprint in equation (4), p. 1272, of the above paper. It should read

$$
H^{1}(Q, \mathbf{C} \backslash\{0\})=\widehat{\pi_{1}(Q)}
$$

More correctly, we should write

$$
H^{1}(Q, \mathbf{C} \backslash\{0\})=\operatorname{Hom}\left(\pi_{1}(Q), \mathbf{C} \backslash\{0\}\right)
$$

The characters of $\pi_{1}(Q)$ are defined to be only the bounded one-dimensional representations which are in one-to-one correspondence to the elements of $H^{1}(Q, \mathrm{U}(1))$. Note that in general the constant-coefficient cohomology groups $H^{1}(Q, \mathrm{C} \backslash\{0\})$ and $H^{1}(Q, \mathrm{U}(1))$ are not the same. Hence, one should replace $C \backslash\{0\}$ by $\mathbf{U}(1)$ in the formulas and, accordingly, $\operatorname{GL}(n, C)$ by $\mathbf{U}(n)$ (cf. Section 2.1.4). However, the essence of the statements in the paper does not change.

Remark. Whether one defines a pure state as a unit ray or as a ray is a matter of taste for a globally defined wave function. But if $\pi_{1}(Q)$ is a group containing elements, which does not have finite order, then we will get norm obstructions (as opposed to the investigated phase obstructions) that are ignored in the paper but possibly give a new physics. These obstructions occur, for example, in the familiar case $\pi_{1}(Q)=\mathbf{Z}$ and in the exotic case $\pi_{1}(Q)=\mathbf{R}$ (Araki et al., 1971). They are labeled by a continuous real parameter and may be dynamically interpolated, since the smooth function valued cohomologies classifying the dynamics coincide.

The first two words in line 4, p. 1280, should read, of course: fractional statistics. Figure 2 should be supplemented by the following drawing in order to show the order-two property.

Finally, I neglected to cite my own Ph.D. thesis (in German; Hamburg University, 1987), on part of which the paper is based.

\footnotetext{
${ }^{1}$ This paper originally appeared in International Journal of Theoretical Physics, 28, 1269-1310 (1989).

${ }^{2}$ II. Institut für Theoretische Physik und I. Institut für Theoretische Physik, University of Hamburg, D-2000 Hamburg 36, Federal Republic of Germany.
} 
Figure 2 should be supplemented by the following drawing in order to show the order-two property.

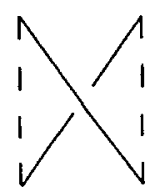

1

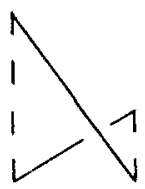

2

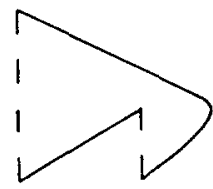

3

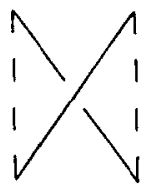

4

Finally, I neglected to cite my own Ph.D. thesis (in German; Hamburg University, 1987), on part of which the paper is based. 